\title{
Probing the size of a magnetosphere of a young solar-like star
}

\author{
Catrina M. Hamilton ${ }^{1}$, C. M. Johns-Krull ${ }^{2}$, R. Mundt ${ }^{3}$, \\ W. Herbst ${ }^{4}$ and J.N. Winn ${ }^{5}$ \\ ${ }^{1}$ Department of Physics and Astronomy, Dickinson College, Carlisle, PA 17013, USA \\ email: hamiltoc@dickinson.edu \\ ${ }^{2}$ Department of Physics and Astronomy, Rice University, Houston, TX 77005, USA \\ email: cmj@rice.edu \\ ${ }^{3}$ Max-Planck-Institut für Astronomie, Königstuhl 17, D-69117 Heidelberg, Germany \\ email: mundt@mpia.de \\ ${ }^{4}$ Astronomy Department, Wesleyan University, Middletown, CT 06459, USA \\ email: wherbst@wesleyan.edu \\ ${ }^{5}$ Department of Physics, Massachusetts Institute of Technology, Cambridge, MA 02139, USA \\ email: jwinn@space.mit.edu
}

\begin{abstract}
We have obtained high resolution spectra of the pre-main sequence binary system $\mathrm{KH}$ 15D (V582 Mon) while the star is fully visible, fully occulted, and during several ingress and egress events over the course of five contiguous observing seasons. The $\mathrm{H} \alpha$ line profile is a standard probe of the magnetospheric accretion flows on young stars such as KH 15D. We use these time series data to map out the size of the magnetosphere and find that it changes size from one observing season to the next.
\end{abstract}

Keywords. Line: profiles - stars: individual (KH 15D, V582 Mon) - stars: pre-main-sequence

\section{Introduction}

$\mathrm{KH} 15 \mathrm{D}$ (V582 Mon) is an eclipsing pre-main-sequence binary system with a period of 48.37 days and an eccentric orbit (Hamilton et al. 2005). The eclipses occur whenever the motion of a star carries it behind a circumbinary ring (Chiang \& Murray-Clay 2004; Winn et al. 2004). As the edge of the occulting matter cuts across the visible star and its magnetosphere, it acts like a "natural coronagraph", progressively covering or revealing structure within a few stellar radii of the photosphere (Hamilton et al. 2003).

\section{The $\mathbf{H} \alpha$ Emission Line Profiles}

Out of eclipse (large $+\mathrm{R}$ ), one sees a redshifted absorption component in the $\mathrm{H} \alpha$ line profile that extends below the stellar continuum. During eclipse, the line profile is narrower and double-peaked, with an underlying broad component, believed to arise in the magnetosphere, which ranges from about -350 to $+350 \mathrm{~km} / \mathrm{s}$. The profiles that have "shoulders" are considered evidence for magnetospheric accretion (see profiles in Figures 1 and 2). From these profiles and the distance the star is from the screen at each epoch, we infer that the magnetosphere is changing in size. 

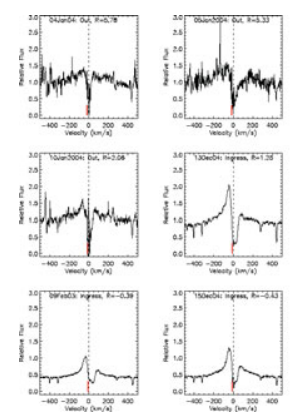
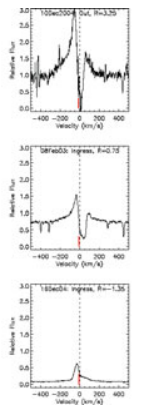
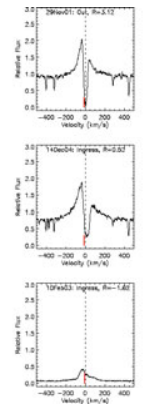
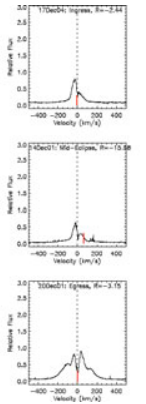
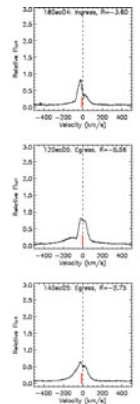
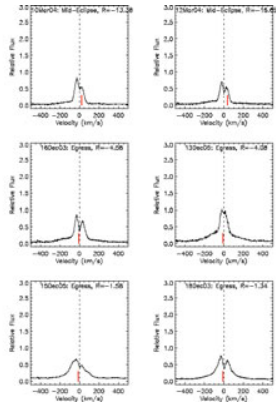

Figure 1. $\mathrm{H} \alpha$ emission line profiles.

\section{Magnetospheric Toy Model for $\mathbf{H} \alpha$}

A simple dipole model is computed (Alencar et al. (2001)) where material freely falls from a disk to the star along field lines. We consider a model that employs a linear flux distribution and includes rotation. We vary the inclination and match the output to an out-of-eclipse profile. We then vary the distance that the star is from the screen and scale the model profile by the photometry appropriate for that night. Our results are shown in Figure 3. In all cases, the inclination was set to $15^{\circ}$.
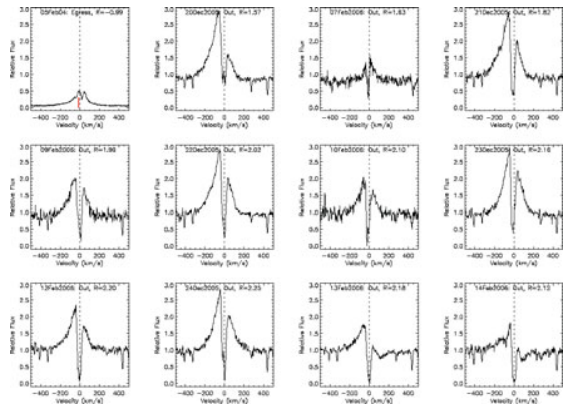

Figure 2. $\mathrm{H} \alpha$ emission line profiles.
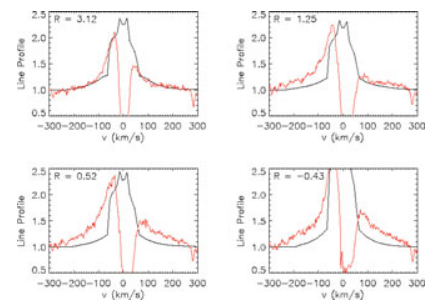

Figure 3. $\mathrm{H} \alpha$ line profiles as calculated by a magnetospheric toy model employed by Alencar et al. (2001) to model the broad wings observed in the $\mathrm{KH} 15 \mathrm{D} \mathrm{H} \alpha$ line profiles, which are believed to arise in the magnetosphere.

\section{Conclusions}

Our spectroscopic studies have revealed intrinsic variability and amazing structure in the $\mathrm{H} \alpha$ line profiles caused by high velocity gas flows in the vicinity of the stars. As the model profile is eclipsed, the shape is generally consistent with the observed profile, however, the flux level does not match the observations. We intend to further investigate the influence the many geometrical parameters and different flux distributions has on the model profiles.

\section{References}

Alencar, S. H. P, Johns-Krull, C. M., \& Basri, G. 2001, AJ 122, 3335

Chiang, E. I. \& Murray-Clay, R. A. 2004, ApJ 607, 913

Hamilton, C. M., et al. 2003, ApJ 591, L45

Hamilton, C. M., et al. 2005, AJ 130, 1896

Winn, J. N., et al. 2004, ApJ 603, L45 